\section{La mala calidad de sueño se asocia a una menor adherencia al tratamiento antirretroviral de gran actividad en pacientes peruanos con infección por VIH/SIDA}

\author{
Poor quality of sleep associated with low \\ adherence to highly active antiretroviral \\ therapy in Peruvian patients with HIV/AIDS
}

\author{
A má qualidade do sono associada à menor \\ aderência à terapia antirretroviral de alta \\ atividade em pacientes peruanos \\ com infecção pelo HIV/AIDS
}

Jorge Renzo Tello-Velásquez 1 Bruno Eduardo Díaz-Llanes 1 Edward Mezones-Holguín 1,2 Alfonso J. Rodríguez-Morales 3 Charles Huamaní 2

Adrián V. Hernández 1 Jorge Arévalo-Abanto 4

\footnotetext{
${ }_{1}$ Escuela de Medicina, Universidad Peruana de Ciencias Aplicadas, Lima, Perú.

2 Centro Nacional de Salud Pública, Instituto Nacional de Salud, Lima, Perú. 3 Facultad de Ciencias de la Salud, Universidad Tecnológica de Pereira, Pereira, Colombia. 4 Servicio de Infectología, Hospital Nacional Dos de Mayo, Lima, Perú.

Correspondencia B. E. Díaz-Llanes Escuela de Medicina, Universidad Peruana de Ciencias Aplicadas. Calle San Ignacio 198, Dpto 401, Santiago de Surco, Lima, LIMA33, Perú.

brunodiazllanes@gmail.com
}

\begin{abstract}
This cross-sectional study analyzed the association between poor quality of sleep and adherence to highly active antiretroviral therapy (HAART) in 389 Peruvian patients with HIVIAIDS. Poor quality of sleep was measured with the Pittsburgh Sleep Quality Index (PSQI) and adherence with the CEAT-VIH (Peruvian adaptation). A Poisson generalized linear model with robust standard errors was used to estimate prevalence ratios and 95\%CI. A crude model showed that mild, moderate, and severe poor quality of sleep were associated with inadequate treatment adherence. In the adjusted model for variables associated in the bivariate analysis or variables theoretically associated with adherence, only moderate/severe poor quality of sleep remained associated $(P R=1.34,95 \% C I: 1.17-1.54 ;$ and $P R=1.34,95 \% C I: 1.16-1.57$, respectively). The study concluded that moderate/severe poor qual ity of sleep was independently associated with adherence to HAART. Assessing quality of sleep may be helpful in the comprehensive evaluation of HIV patients.
\end{abstract}

Sleep Disorders; HIV; Highly Active Antiretroviral Therapy

\section{Resumen}

Con el objetivo de evaluar si existe asociación entre la mala calidad de sueño y la adherencia al tratamiento antirretroviral de gran actividad (TARGA) en personas con infección por VIH/ SIDA; llevamos a cabo un estudio analítico de corte transversal que incluyó 389 pacientes peruanos en TARGA. La mala calidad de sueño fue medida con la Escala de Calidad de Sueño de Pittsburgh y la adherencia con el CEAT-VIH. Realizamos un modelo lineal generalizado de familia Poisson, con errores estándar robustos para estimar razones de prevalencia y su IC95\%. A nivel crudo la mala calidad de sueño leve, moderada y severa se asoció a la adherencia inadecuada. Al ajustar por las variables asociadas en el análisis bivariado o por las variables teóricamente asociadas a la adherencia, sólo la mala calidad de sueño moderada/severa se mantuvo asociada (RP = 1,34; IC95\%: 1,17-1,54 y $R P=1,34$; IC95\%: 1,16-1,57; respectivamente). Se concluye que la mala calidad de sueño moderada/severa se asocia de manera independiente con la adherencia al TARGA. La evaluación de la calidad de sueño podría por ende ser útil en la valoración integral de los pacientes con VIH.

Trastornos del Sueño; VIH; Terapia

Antirretroviral Altamente Activa 


\section{Introducción}

La infección por el virus de inmunodeficiencia humana (VIH) y el síndrome de inmunodeficiencia adquirida (SIDA) constituyen un problema de salud pública global 1,2,3,4,5,6. En 2013 en el mundo se reportaron 1,8 millones de casos de infección por el VIH con una prevalencia de 29,2 millones de personas viviendo con VIH 1. En Perú, para ese mismo año se estimaron 65.000 personas viviendo con VIH y 2.800 muertes por SIDA 4,5 . El control, manejo y prevención de esta enfermedad requiere una importante inversión de recursos públicos para su prevención, control y tratamiento, especialmente en países de ingresos bajos o medianos 6 . El tratamiento antirretroviral de gran actividad (TARGA) es una de las más importantes medidas para evitar las enfermedades oportunistas y las muertes asociadas 3,7,8,9,10.

Gracias a la amplia cobertura de TARGA en pacientes con VIH/SIDA, esta condición se reconoce como una entidad de manejo crónico, a nivel mundial, así como en Perú 11,12,13,14,15. En este contexto, la adherencia a la indicación terapéutica por parte de los pacientes constituye un factor fundamental para el éxito o fracaso del TARGA $16,17,18$. Sin embargo, la adherencia se ve afectada por múltiples factores, en particular por los efectos secundarios del tratamiento 17,18,19,20. Problemas de salud mental como la depresión 21, así como el consumo de alcohol 22,23 y el abuso de sustancias, se asocian también a una baja adherencia al tratamiento 20,24,25,26,27,28,29. Además, se ha encontrado asociación con efectos adversos específicos de los antirretrovirales 30,31, entre otras causas 32,33 .

Entre los efectos asociados al TARGA se viene explorando su potencial para afectar la calidad del sueño, explorado en varias series de pacientes con VIH 34,35,36,37, donde ello se reporta como una importante barrera de adherencia al TARGA. La mala calidad de sueño se encuentra asociada a problemas de concentración, alteraciones en recordar la toma de medicamentos, el estado de ánimo, entre otras; lo cual se asocia a una menor adherencia $38,39,40,41$. No obstante, a pesar de la literatura mencionada, esta asociación ha sido escasamente estudiada en población latinoamericana.

Por ello, nuestro estudio tuvo como objetivo evaluar si existe asociación entre la calidad de sueño y la adherencia al TARGA, controlado por variables demográficas y clínicas, en pacientes peruanos infectados por el VIH. Estos resultados podrían servir para remarcar la necesidad de la evaluación del sueño como problema frecuente en pacientes con VIH, pero sobre todo como un factor adicional que influye en la adherencia a los regímenes terapéuticos farmacológicos en dicha población.

\section{Métodos}

\section{Diseño y lugar de estudio}

Llevamos a cabo un estudio analítico de corte transversal durante los años 2012 y 2013 en la consulta ambulatoria del Servicio de Infectología del Hospital Nacional Dos de Mayo, Lima, Perú. Se seleccionó este hospital por ser un centro de referencia nacional de Ministerio de Salud de Perú (MINSA) y por realizar evaluaciones integrales de los pacientes atendidos con infección por VIH. Este nosocomio atiende en promedio sobre 320.000 pacientes por año en consultas externas.

\section{Población, muestra y muestreo}

Incluimos personas adultas de 18 a 60 años con diagnóstico de infección por VIH en TARGA. Excluimos a pacientes con afasia, desorden cerebro vascular con secuelas, retardo mental, neoplasia maligna y en medicación con benzodiacepinas $\mathrm{o}$ antidepresivos.

Estimamos un tamaño de muestra mínimo de 381 pacientes, basado en lo necesario para un modelo de regresión de desenlace dicotómico, considerando un nivel de confianza del 95\% para una prueba a dos colas, con una potencia estadística de $80 \%$ y un coeficiente $\beta=0,507$ $(\mathrm{OR}=1,66){ }^{41}$ y una proporción de mala adherencia del $79 \% 37$. En base al estudio piloto, calculamos un $1 \%$ de fichas inconclusas y $10 \%$ de proporción de rechazo, por lo que se requería invitar a participar a 428 pacientes. Para el cálculo de tamaño de muestra usamos nQuery Advisor versión 6.0 (Statistical Solutions, Cork, Irlanda).

La selección de los pacientes se realizó de manera aleatoria simple, considerando el listado de atención diario del Hospital Nacional Dos de Mayo.

\section{Variables y mediciones}

Construimos un cuestionario auto-aplicado conformado por cuatro secciones: datos sociodemográficos, aspectos clínicos, calidad de sueño (variable de exposición) y adherencia al TARGA (variable de respuesta) 42,43,44,45,46,47,48.

\section{- Datos sociodemográficos}

Medimos la edad (en años), el sexo (hombre/ mujer), la orientación sexual (heterosexual, homosexual, bisexual), el grado de instrucción (pri- 
maria, secundaria, superior no universitario, superior universitario), estado civil (pareja estable: casado, conviviente; sin pareja estable: soltero, viudo y divorciado), religión (católica, evangélica, judía, otra, agnóstico o ateo), actividad laboral (empleado, obrero, jubilado, ama de casa, comerciante, estudiante/otro/desempleado) y número de trabajos (ninguno, un trabajo, más de un trabajo).

\section{- Aspectos clínicos}

Consignamos el tiempo con diagnóstico de infección por VIH (en meses), tiempo en tratamiento antirretroviral (en meses), carga viral (detectable o indetectable), recuento de CD4 $(<200$ células/ $\mathrm{mL} o \geq 200$ células/mL) y presencia de comorbilidades (no o sí: diabetes mellitus, hipertensión arterial, artritis reumatoide, artrosis, tuberculosis, enfermedad pulmonar obstructiva crónica, asma, otros)

\section{- Calidad de sueño}

Utilizamos la Escala de Calidad de Sueño de Pittsburgh (ECSP) validada al español 42 , la cual posee una buena consistencia interna ( $\alpha$ de Cronbach $=$ 0,78 ), una sensibilidad de $88,63 \%$ y una especificidad de 74,99\% para detectar trastornos del sueño 42. La ECSP consta de 19 ítems dentro de siete componentes evaluados: la calidad subjetiva del sueño, la latencia de sueño, la duración del sueño, la eficiencia habitual del sueño, las perturbaciones del sueño, el uso de medicación hipnótica, y la disfunción diurna 42 .

La puntuación global de la ECSP permite clasificar la calidad del sueño en cuatro categorías: buena calidad de sueño $(\leq 5)$, mala calidad del sueño leve (6-8), mala calidad del sueño moderada (9-11) y mala calidad del sueño severa (> 11) 42,43,44. Sin embargo, para el análisis de la asociación con la adherencia al TARGA, las malas calidad del sueño moderada y severa fueron incluidas en la misma categoría, puesto que en ambos casos se requiere de evaluación médica especializada 43 . Esta escala ha sido previamente usada en varios estudios sobre población peruana, presentando buena aceptabilidad por parte de los entrevistados 49,50,51.

\section{- Adherencia al TARGA}

Empleamos la versión validada al español del Cuestionario de Evaluación de la Adhesión al Tratamiento Antirretroviral (CEAT-VIH). Este instrumento tiene una buena consistencia interna ( $\alpha$ de Cronbach $=0,7$ ); así como una correlación positiva significativa entre la puntuación global con el recuento de CD4 ( $\mathrm{r}=0,439 ; \mathrm{p}<0,005)$ y una correlación negativa significativa con la carga viral $(\mathrm{r}=-0,548 ; \mathrm{p}<0,005)$, el cual nos permitió clasificar la adherencia mediante 20 ítems, cuya puntuación individual va entre 0 a 1 y 1 a 5 , con puntuaciones globales que oscilan entre 17 y 89 . En base a la puntuación global se generan cuatro categorías: baja adherencia ( $\leq 73$ puntos), adherencia insuficiente (74-80 puntos), adherencia adecuada (81-85 puntos) y adherencia estricta (> 85 puntos) 45 . No obstante, cuando se evaluó la asociación con otras variables, se generó una variable dicotómica a partir de la clasificación original: (1) adecuada adherencia, compuesta por las categorías suficiente y estricta; y (2) inadecuada adherencia, refiriéndose a las categorías baja e insuficiente 45,46. El CEAT-VIH ha sido usado en diversas investigaciones en diversas regiones del mundo, incluyendo estudios llevados a cabo en Perú 47,48. Se contó con la autorización del autor de la escala original.

\section{Procedimientos}

\section{- Prueba piloto}

Realizamos una prueba piloto en 23 pacientes en enero 2012. La selección de los participantes fue por conveniencia. Estimamos los tiempos promedio de respuesta e hicimos reformulaciones a algunas preguntas del cuestionario 52 . Los pacientes que participaron del estudio piloto no fueron incluidos en el estudio final.

\section{- Recolección de datos}

Los participantes fueron contactados a la salida de la consulta ambulatoria en los consultorios externos del Servicio de Infectología del Hospital. Tras la selección realizada de los listados de atención, explicamos los objetivos de la investigación y les invitamos a participar en el estudio. El cuestionario fue autoaplicado y se entrenaron estudiantes de medicina para solucionar las dudas y facilitar las respuestas de los participantes.

\section{Análisis estadístico}

\section{- Generación de la base de datos}

Generamos una base de datos en Microsoft Excel 2010 (Microsoft Corp., EEUU), mediante digitación ciega e independiente por dos personas. Posteriormente, exportamos la base al software Stata versión 11.0 (Stata Corp., College Station, Texas, EEUU) para realizar el análisis de datos. Aleatoriamente, seleccionamos 20 observaciones, en las cuales se evaluó la concordancia entre 
la digitación y el registro físico en fichas. Asimismo, se evaluó la proporción de acuerdo entre los digitadores.

\section{- Estadística descriptiva}

Para las variables categóricas usamos frecuencias absolutas y relativas. Para las variables numéricas se utilizaron medias y desviación estándar.

\section{- Estadística inferencial}

A nivel bivariado para evaluar la asociación entre la adherencia y la calidad de sueño, así como otras variables categóricas, realizamos pruebas de significancia estadística por medio de chi cuadrado y el test exacto de Fisher (dependiendo de los valores esperados). Para evaluar la asociación entre adherencia y variables numéricas, utilizamos la prueba t de Student para muestras independientes, previa evaluación de los supuestos de normalidad y homogeneidad de varianzas, con los test de Shapiro Wilk y Levene, respectivamente.

Se realizaron tres modelos de regresión: un bivariado (crudo), un modelo multivariado ajustado por las variables significativas en el bivariado (modelo ajustado 1) y un modelo multivariado, ajustado por las variables que teóricamente están asociadas a la adherencia (modelo ajustado 2). En todos los casos reportamos razones de prevalencia (RP) con sus respectivos intervalos de 95\% de confianza (IC95\%), a partir de modelos lineales generalizados de familia Poisson con errores estándar robustos, en los cuales la adherencia al TARGA -como variable de respuesta dicotómica- era la variable de respuesta y la calidad de sueño era la variable de exposición.

\section{Aspectos éticos}

El protocolo fue aprobado por el Comité de Ética de la Universidad Peruana de Ciencias Aplicadas, Lima, Perú (CEI/173-11-13) y la Oficina de Apoyo a la Capacitación, Docencia e Investigación del Hospital Nacional Dos de Mayo, Lima, Perú (Registro no 00387). Nuestro estudio cumplió con los principios éticos contenidos en la Declaración de Helsinki 53. A todos los pacientes convocados se les proporcionó alcances del estudio y se les invitó a firmar de manera voluntaria el consentimiento informado.

\section{Resultados}

\section{Selección de participantes}

Invitamos a un total de 436 pacientes a participar del estudio. De ellos, 35 pacientes no desearon ser parte de la investigación (proporción de rechazo: $8 \%$ ). Se eliminaron 12 observaciones que no habían resuelto la totalidad de ítems para la variable de exposición y respuesta. Finalmente, incluimos 389 pacientes en el análisis (Figura 1).

\section{Análisis descriptivo}

Aproximadamente dos tercios de los pacientes eran varones (263) (Tabla 1), la media de la edad fue de 35,3 años ( \pm desviación estándar de 10,8 años). Cerca de la mitad de los encuestados eran empleados en un centro de trabajo y 6,9\% eran desempleados (Tabla 1). Más de la mitad de los pacientes tenía menos de 5 años de diagnóstico de VIH (media 55,2 meses \pm 45,3 meses) (Tabla 2). Siete de cada diez pacientes tenía menos de cinco años en TARGA (media 43,8 meses $\pm 36,9$ meses) (Tabla 2). El esquema de TARGA más utilizado fue el conformado por dos inhibidores nucleósidos y un inhibidor no nucleósido de la transcriptasa reversa (2 INTR+1 INNTR). Casi la totalidad de pacientes presentaba una carga viral indetectable o niveles de CD4 mayores a 200 células/ $\mathrm{mL}$. Fueron 62 pacientes los que reportaron al menos alguna comorbilidad; diabetes mellitus $(3,1 \%)$ y asma $(2,6 \%)$ fueron las más frecuentes. El 60,7\% de los pacientes presentaba algún grado de mala calidad del sueño, teniendo la mala calidad del sueño moderada la mayor frecuencia al ser encontrada en 128 encuestados. El 74,5\% de los pacientes tenían adherencia inadecuada y el 25,5\% tenían adherencia adecuada (Tabla 2).

\section{Análisis bivariado}

La adherencia adecuada fue significativamente mayor con mejores grados de instrucción (superior) ( $p=0,018$ ), el esquema de tratamiento TARGA ( $p=0,036$ ), la presencia de comorbilidades $(\mathrm{p}=0,016)$ y la calidad de sueño $(\mathrm{p}<0,001)$ Por otro lado, el género, la orientación sexual, la religión, la actividad laboral, el número de trabajos, el nivel de CD4 y el tener una pareja estable no tuvieron asociación estadísticamente significativa con la adherencia. Además, la edad, el tiempo de TARGA y el tiempo de diagnóstico de VIH -tanto en el análisis como variables numéricas como categóricas- no se asociaron con la adherencia (Tabla 3). Asimismo, en el análisis de tendencia se evidenció una asociación estadísticamente significativa en la proporción de sujetos 


\section{Figura 1}

Flujograma de la participación de pacientes en el estudio.

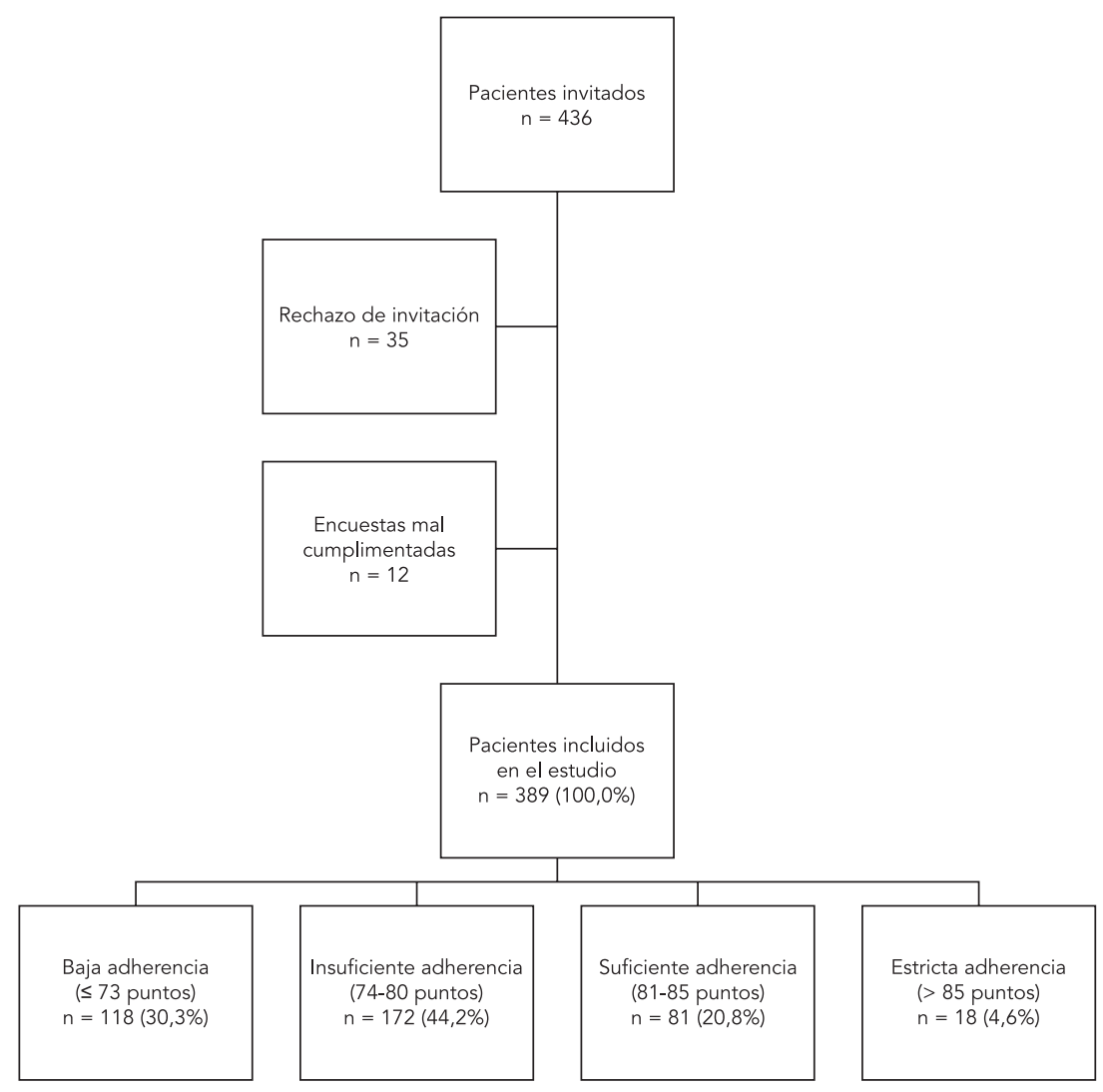

con adherencia inadecuada por cada nivel de deterioro de la calidad del sueño.

\section{Modelos de regresión}

En el modelo crudo observamos que la mala calidad del sueño leve (RP = 1,2; IC95\%: 1,01- 1,42) y mala calidad del sueño moderada/severa (RP = 1,37; IC95\%: 1,19-1,57) se asocian a una adherencia inadecuada. Asimismo, el tener grado de instrucción primaria $(\mathrm{RP}=1,28$; IC95\%: 1,09$1,5)$, el esquema basado en un inhibidor de la proteasa y dos inhibidores nucleósidos de la transcriptasa reversa $(\mathrm{RP}=1,78$; IC95\%: 1,01 $3,15)$ y el tener alguna comorbilidad $(\mathrm{RP}=1,21$; IC95\%: 1,07-1,36) se asocian con la adherencia inadecuada (Tabla 4).

El modelo ajustado 1 muestra que la mala calidad del sueño moderada/severa de la calidad del sueño se asocia a una adherencia inadecua- da $(\mathrm{RP}=1,34$; IC95\%: 1,17-1,54). El nivel de instrucción primaria ( $\mathrm{RP}=1,33$; IC95\%: 1,13-1,57) y el tener alguna comorbilidad también se mantienen asociadas (RP = 1,15; IC95\%: 1,06-1,30). En el modelo ajustado 2 se observa que la mala calidad del sueño moderada/severa y la adherencia inadecuada se mantienen asociados (RP = 1,34; IC95\%: 1,16-1,55). Adicionalmente, el grado de instrucción primaria (RP = 1,33; IC95\%: 1,131,55) y la presencia de comorbilidades persisten asociados. Como puede observarse en ambos modelos ajustados, la mala calidad del sueño leve ya no presenta evidencia estadística de asociación con la adherencia al TARGA. Finalmente, puede observarse que la probabilidad de tener una adherencia inadecuada, cuando se tiene mala calidad del sueño moderada/severa, es 1,37 veces la probabilidad de tener una adherencia inadecuada cuando se tiene una buena calidad del sueño en ambos modelos ajustados. 
Tabla 1

Características generales de la población en estudio $(\mathrm{N}=389)$.

\begin{tabular}{|c|c|c|}
\hline Variable & $\mathrm{n}$ & $\%$ \\
\hline \multicolumn{3}{|l|}{ Edada (años) } \\
\hline $18-29$ & 138 & 35,5 \\
\hline $30-39$ & 129 & 33,2 \\
\hline $40-49$ & 73 & 18,8 \\
\hline $50-60$ & 49 & 12,5 \\
\hline \multicolumn{3}{|l|}{ Género } \\
\hline Varón & 263 & 67,6 \\
\hline Mujer & 126 & 32,4 \\
\hline \multicolumn{3}{|l|}{ Orientación sexual } \\
\hline Heterosexual & 258 & 66,3 \\
\hline Homosexual & 102 & 26,2 \\
\hline Bisexual & 29 & 7,5 \\
\hline \multicolumn{3}{|l|}{ Grado de instrucción } \\
\hline Primaria & 86 & 22,1 \\
\hline Secundaria & 194 & 49,9 \\
\hline Superior no universitaria & 47 & 12,1 \\
\hline Superior universitaria & 62 & 15,9 \\
\hline \multicolumn{3}{|l|}{ Pareja estable } \\
\hline Con pareja estable & 282 & 72,5 \\
\hline Sin pareja estable & 107 & 27,5 \\
\hline \multicolumn{3}{|l|}{ Religión } \\
\hline Católica & 309 & 79,4 \\
\hline Evangélica & 46 & 11,8 \\
\hline Judía & 2 & 0,5 \\
\hline Otra & 27 & 6,0 \\
\hline Agnóstico & 5 & 1,3 \\
\hline \multicolumn{3}{|l|}{ Actividad laboral } \\
\hline Empleado & 183 & 47,1 \\
\hline Obrero & 30 & 7,7 \\
\hline Jubilado & 7 & 1,8 \\
\hline Ama de casa & 45 & 11,6 \\
\hline Comerciante & 41 & 10,5 \\
\hline Estudiante & 34 & 8,7 \\
\hline Otros & 22 & 5,7 \\
\hline Desempleado & 27 & 6,9 \\
\hline \multicolumn{3}{|l|}{ Problemas de alcohol } \\
\hline Sin problemas & 282 & 72,5 \\
\hline Con problemas & 75 & 19,3 \\
\hline Dependencia & 32 & 8,2 \\
\hline
\end{tabular}


Tabla 2

Características de la infección por VIH/SIDA de la población en estudio ( $\mathrm{N}=389)$.

\begin{tabular}{|c|c|c|}
\hline Variable & $\mathrm{n}$ & $\%$ \\
\hline \multicolumn{3}{|l|}{ Comorbilidades } \\
\hline Sin comorbilidad & 327 & 84,1 \\
\hline Con comorbilidad & 62 & 15,9 \\
\hline \multicolumn{3}{|l|}{ Tiempo de diagnóstico de $\mathrm{VIH}$ (años) } \\
\hline$<1$ & 76 & 19,5 \\
\hline $1-5$ & 189 & 48,6 \\
\hline $5-10$ & 101 & 26,0 \\
\hline$>10$ & 23 & 5,9 \\
\hline \multicolumn{3}{|l|}{ Tiempo con tratamiento TARGA (años) } \\
\hline$<1$ & 111 & 28,5 \\
\hline $1-5$ & 185 & 47,5 \\
\hline $5-10$ & 85 & 21,8 \\
\hline$>10$ & 8 & 2,0 \\
\hline \multicolumn{3}{|l|}{ Carga viral } \\
\hline Indetectable & 373 & 95,9 \\
\hline Detectable & 16 & 4,1 \\
\hline \multicolumn{3}{|l|}{ Nivel de CD4 (células/mL) } \\
\hline$<200$ & 6 & 1,5 \\
\hline$\geq 200$ & 383 & 98,5 \\
\hline \multicolumn{3}{|l|}{ Esquema de tratamiento TARGA } \\
\hline 3 INRT * & 15 & 3,9 \\
\hline $1 \mathrm{IP}+2 \mathrm{INTR} * \star$ & 24 & 6,2 \\
\hline 2 INRT + 1 NNTR *** & 350 & 89,9 \\
\hline \multicolumn{3}{|l|}{ Calidad de sueño } \\
\hline Buena calidad del sueño & 153 & 39,3 \\
\hline Mala calidad de sueño leve & 85 & 21,9 \\
\hline Mala calidad de sueño moderada & 128 & 32,9 \\
\hline Mala calidad de sueño severa & 23 & 5,9 \\
\hline \multicolumn{3}{|l|}{ Adherencia } \\
\hline Baja & 118 & 30,3 \\
\hline Insuficiente & 172 & 44,2 \\
\hline Suficiente & 81 & 20,8 \\
\hline Estricta & 18 & 4,7 \\
\hline
\end{tabular}

TARGA: tratamiento antirretroviral de gran actividad.

* 3 INTR = tres inhibidores nucleósidos de la transcriptasa reversa;

** 1 IP + 2 INTR = un inhibidor de proteasa, más dos inhibidores nucleósidos de la transcriptasa reversa;

*** 2 INTR + 1 NNTR = dos inhibidores nucleósidos de la transcriptasa reversa, más un inhibidor no nucleósido de

la transcriptasa reversa. 
Tabla 3

Análisis bivariado entre la adherencia al TARGA y variables de exposición de la población de estudio ( $N=389$ ).

\begin{tabular}{|c|c|c|c|}
\hline Variable & Adherencia adecuada & $\begin{array}{l}\text { Adherencia } \\
\text { inadequada }\end{array}$ & Valor de $p$ \\
\hline Edad (años)* & $36,24 \pm 11,45$ & $34,98 \pm 10,86$ & 0,26 \\
\hline $18-29$ & $32(23,2)$ & $106(76,8)$ & \\
\hline $30-39$ & $31(24,0)$ & $98(75,0)$ & \\
\hline $40-49$ & $23(31,5)$ & $50(68,5)$ & 0,51 \\
\hline $50-60$ & $13(26,5)$ & $36(73,5)$ & \\
\hline Género & & & 0,13 \\
\hline Varón & $73(27,8)$ & $190(72,2)$ & \\
\hline Mujer & $26(20,6)$ & $100(79,4)$ & \\
\hline Orientación sexual & & & 0,81 \\
\hline Heterosexual & $68(26,4)$ & $190(73,6)$ & \\
\hline Homosexual & $25(24,5)$ & $77(75,5)$ & \\
\hline Bisexual & $6(20,7)$ & $23(79,3)$ & \\
\hline Grado de instrucción & & & 0,018 \\
\hline Primaria & $12(13,9)$ & $74(86,1)$ & \\
\hline Secundária & $51(26,3)$ & $143(73,7)$ & \\
\hline Superior no universitaria & $16(34,0)$ & $31(66,0)$ & \\
\hline Superior universitaria & $20(32,3)$ & $42(67,7)$ & \\
\hline Pareja estable & & & 0,194 \\
\hline Con pareja estable & $77(27,3)$ & $205(72,7)$ & \\
\hline Sin pareja estable & $22(20,6)$ & $85(79,4)$ & \\
\hline Religión & & & 0,63 \\
\hline Católica & $84(27,2)$ & $225(72,8)$ & \\
\hline Evangélica & $8(17,4)$ & $38(82,6)$ & \\
\hline Judía & $0(0,0)$ & $2(100,0)$ & \\
\hline Otra & $6(22,2)$ & $21(77,8)$ & \\
\hline Agnóstico & $1(20,0)$ & $4(80,0)$ & \\
\hline Número de trabajos & & & 0,13 \\
\hline Sin trabajo & $9(32,1)$ & $19(67,9)$ & \\
\hline 1 & $88(24,6)$ & $270(75,4)$ & \\
\hline 2 o más & $2(66,7)$ & $1(33,3)$ & \\
\hline Comorbilidades & & & 0,16 \\
\hline $\operatorname{Sin}$ & $91(27,8)$ & $236(72,8)$ & \\
\hline Con & $8(12,9)$ & $54(87,1)$ & \\
\hline Problemas de alcohol & & & 0,68 \\
\hline $\operatorname{Sin}$ & $64(2,7)$ & $218(77,3)$ & \\
\hline Con & $27(36,0)$ & $48(64,0)$ & \\
\hline Dependencia & $8(25,0)$ & $24(75,0)$ & \\
\hline Tiempo de diagnóstico de VIH (años) * & $57,8 \pm 4,2$ & $54,3 \pm 44,3$ & 0,5 \\
\hline$<1$ & $19(25,0)$ & $57(75,0)$ & 0,5 \\
\hline $1-5$ & $49(25,9)$ & $140(74,1)$ & \\
\hline $5-10$ & $23(22,8)$ & $78(77,2)$ & \\
\hline$>10$ & $8(34,8)$ & $15(65,2)$ & \\
\hline Tiempo com tratamiento TARGA (año) * & $47,3 \pm 37,9$ & $42,2 \pm 36,8$ & 0,22 \\
\hline$<1$ & $25(22,5)$ & $86(77,5)$ & \\
\hline $1-5$ & $47(25,4)$ & $138(74,6)$ & \\
\hline $5-10$ & $24(28,2)$ & $61(71,8)$ & 0,63 \\
\hline$>10$ & $3(37,5)$ & $5(62,5)$ & \\
\hline
\end{tabular}

(continúa) 
Tabla 3 (continuación)

\begin{tabular}{|c|c|c|c|}
\hline Variable & Adherencia adecuada & $\begin{array}{l}\text { Adherencia } \\
\text { inadequada }\end{array}$ & Valor de $p$ \\
\hline Esquea de tratamiento TARGA & & & 0,036 \\
\hline 3 INTR ** & $8(53,3)$ & $7(46,7)$ & \\
\hline $1 \mathrm{IP}+2$ INNTR *** & $4(16,7)$ & $20(83,3)$ & \\
\hline 2 INTR + 1 NNTR \# & $87(24,9)$ & $263(75,1)$ & \\
\hline Calidad de sueño & & & $<0,001$ \\
\hline Buena calidad del sueño & $57(37,3)$ & $96(62,7)$ & \\
\hline Mala calidad de sueño leve & $21(24,7)$ & $64(75,3)$ & \\
\hline Mala calidad de sueño moderada & $21(16,4)$ & $107(83,6)$ & \\
\hline Mala calidad de sueño severa & $0(0,0)$ & $23(100,0)$ & \\
\hline
\end{tabular}

TARGA: tratamiento antirretroviral de gran actividad.

* Media \pm desviación estándar;

** 3 INTR = tres inhibidores nucleósidos de la transcriptasa reversa;

*** 1 IP + 2 INTR = un inhibidor de proteasa, más dos inhibidores nucleósidos de la transcriptasa reversa;

\# 2 INTR + 1 NNTR = dos inhibidores nucleósidos de la transcriptasa reversa, más un inhibidor no nucleósido de la

transcriptasa reversa.

Tabel 4

Modelos lineales generalizados de familia Poisson con errores estándar robustos para evaluar la asociación entre la calidad de sueño y la adherencia al TARGA ( $\mathrm{N}=389)$.

\begin{tabular}{|c|c|c|c|c|c|c|}
\hline \multirow[t]{2}{*}{ Variable } & \multicolumn{2}{|c|}{ Modelo crudo } & \multicolumn{2}{|c|}{ Modelo ajustado 1 * } & \multicolumn{2}{|c|}{ Modelo ajustado 2 ** } \\
\hline & RP (IC95\%) & Valor de $p$ & RP (IC95\%) & Valor de p & RP (IC95\%) & Valor de $p$ \\
\hline Calidad de sueño & & & & & & \\
\hline $\begin{array}{l}\text { Buena calidad } \\
\text { del sueño }\end{array}$ & (base) & & (base) & & (base) & \\
\hline $\begin{array}{l}\text { Mala calidad de } \\
\text { sueño leve }\end{array}$ & $1,20(1,01-1,43)$ & 0,038 & $1,16(0,98-1,37)$ & 0,088 & $1,16(0,98-1,38)$ & 0,078 \\
\hline $\begin{array}{l}\text { Mala calidad de } \\
\text { sueño moderada/ } \\
\text { severa }\end{array}$ & $1,37(1,19-1,58)$ & 0,001 & $1,34(1,17-1,55)$ & 0,001 & $1,35(1,17-1,54)$ & 0,001 \\
\hline
\end{tabular}

IC95\%: intervalo de 95\% de confianza; RP: razón de prevalencia; TARGA: tratamiento antirretroviral de gran actividad.

* Modelo ajustado 1: modelo teórico ajustado por grado de instrucción, esquema de tratamiento TARGA, edad, orientación sexual, tiempo con diagnóstico TARGA (meses), tiempo con tratamiento TARGA;

** Modelo ajustado 2: modelo con variables significativas en el análisis bivariado, ajustado por grado de instrucción y esquema de tratamiento TARGA.

\section{Discusión}

Nuestros hallazgos muestran que la mala calidad del sueño moderada/severa se asocia con una adherencia inadecuada al TARGA, de modo independiente a variables clínicas y demográficas. Se observa que la prevalencia de inadecuada adherencia al TARGA es un $34 \%$ mayor en aquellos que tiene mala calidad moderada/severa de la calidad de sueño que en aquellos que tienen buena calidad de sueño. Estos resultados son consis- tentes con lo descrito por otros estudios llevados a cabo en pacientes no latinos en países desarrollados, a pesar de la diferencia en las formas de la medición de adherencia (recuento de pastillas o número de días continuos sin haber tomado el tratamiento) y calidad de sueño (percepción subjetiva) 39,40,54; a pesar de tratarse en algunos casos sólo de mujeres 40,41 .

Se ha descrito que esta asociación podría subyacer en una serie de interacciones conductuales y biológicas, que conllevarían al olvido de 
la toma de medicación, esta situación se produciría también cambios en la rutina diaria, incrementan sus preocupaciones y problemas para el logro de los horarios preestablecidos para la ingesta de medicamentos, donde la somnolencia y los efectos en las actividades diarias pueden jugar un rol central 39,40,41. Otro fenómeno propuesto es que existan medicamentos que pueden llevar a problemas del sueño o haya percepción de ello, y por tanto el paciente deje de tomar los fármacos 54 .

Más aún cuando la de pacientes con mala calidad de sueño es superior a la reportada en la población general, que oscila en el $30 \%$, este hallazgo ha sido reportado también por otros estudios en población VIH 55 y es comparable a lo descrito en poblaciones especiales como los ancianos o los estudiantes de medicina. Esto indicaría de modo indirecto que los pacientes con VIH tienen una mayor frecuencia de mala calidad del sueño.

El nivel de adherencia adecuada encontrado en nuestro estudio es bajo (27\%) y se encuentra en niveles inferiores a lo reportado por tres estudios desarrollados en Sudáfrica: 54,5\%, 49,2\% y $43,2 \% 56,57,58$. Esto podría estar relacionado al instrumento utilizado (CEAT-VIH). Esto nos hace reflexionar en la posibilidad de crear o evaluar nuevas estrategias sanitarias para su mejoría, en las cuales la identificación de la mala calidad del sueño puede ser un factor a considerar.

Otro hallazgo secundario encontrado es la asociación entre adherencia y nivel educacional. Al respecto, estudios previos llevados a cabo han descrito hallazgos similares al de nuestra investigación, al describir la asociación entre el nivel educacional y el grado de adherencia al tratamiento antirretroviral; siendo aquellos que tenían educación primaria o ningún grado de instrucción los menos adherentes 59 . Sin embargo, en otro estudio realizado recientemente 60 , la mayor asociación de frecuencia de niveles bajos de adherencia al TARGA se encontró en las personas que tenían grado de instrucción superior. Esto denota profundizar en estudios relacionados, específicamente, con los aspectos socioeconómicos de la adherencia.

Asimismo, encontramos asociación de la presencia de comorbilidades a la adherencia al TARGA, situación que ha sido descrita en otras series 25,36,61,62. En nuestro estudio no se contó con el número suficiente de casos para tener una frecuencia específica por cada enfermedad que permita incluir en el análisis de asociación. No obstante, como variable genérica la comorbilidad fue incluida en los modelos de regresión ajustados al considerase un confusor importante. Además de ello, se debe comentar que no se pudo evidenciar como en otros estudios, el efecto del alcohol en la calidad del sueño y en la adherencia, pues no fue significativamente asociado 22,23,26,28.

Un tópico de relevancia cuando se discute el tema de adherencia y los problemas relacionados al sueño es el papel que puede jugar la depresión. Puesto que existe una alta asociación entre la adherencia y la depresión en general y en pacientes con VIH 25,26,40,41,61. Asimismo, la alta correlación descrita entre depresión y calidad de sueño, y sobre todo por el hecho que dentro del constructo de depresión incluye una dimensión relacionada a trastornos del sueño, lo que se ve reflejado en la inclusión de ítems dentro de varios de los cuestionarios utilizados para medir depresión 63, ha generado un debate en la academia sobre todo acerca de la direccionalidad de la asociación. Se ha descrito que uno de los mecanismos biológicos más importantes es la desregulación que puede existir del eje hipotálamo-pituitarioadrenal, producto de la duración corta del sueño y a su vez la desregulación puede contribuir a la aparición de síntomas de depresión y ansiedad 40,64,65,66,67. Puesto que la depresión estaría asociada a la mala calidad de sueño y a la adherencia podría ser un factor confusor 67,68 ; sin embargo, si partimos de propuestas previas 40,64, es posible que la depresión se encuentre en la vía causal entre la depresión y al ser producto de los problemas del sueño y conducir a la baja adherencia, situación que ameritaría un análisis de mediación 40 . Al tratarse de un estudio de corte transversal no se efectuó el análisis de mediación -por modelos de ecuaciones estructurales, por ejemplo- o la cadena causal incorporando el correspondiente análisis temporal 67,68. Por ello, nuestro estudio no puede afirmar si el efecto de la calidad de sueño sobre la adherencia puede ser directo o indirecto. En ese sentido, nuestros hallazgos se presentan como asociación simplemente, requiriéndose estudios longitudinales para evaluar causalidad. Es importante remarcar que el objetivo del estudio fue evaluar la asociación, más no determinar una relación causal.

Otras limitaciones a considerar son que la medición de las variables principales se obtuvo a partir de escalas auto-administrada, la ECSP y el CEAT-VIH, que no constituyen el patrón de oro correspondiente. Sin embargo, son escalas ampliamente usadas y validadas en el ámbito latinoamericano, y se recomienda su uso en estudios epidemiológicos como el nuestro.

Otro aspecto que hay que considerar como limitación es la posibilidad de sesgo de selección por tratarse de una muestra de una población hospitalaria; no obstante, es un hospital de re- 
ferencia nacional en un nivel de atención que permite la prescripción del TARGA y la dispensación del mismo. Además, el objetivo primario del estudio es la evaluación de una asociación y no el establecer una prevalencia precisa; más allá de que la frecuencias de exposición y de respuesta pueden variar de lo que se encontraría en un estudio poblacional, el diseño muestral es eficiente y permite la evaluación de una asociación.

Más allá de las limitaciones presentadas, nuestro estudio es relevante, puesto que los problemas del sueño, como la mala calidad, no son evaluados de forma rutinaria en las consultas médicas; pudiendo presentarse como efecto adverso al tratamiento, o asociada a otras circunstancias sociales o culturales, que terminan condicionando una baja o discontinuidad del tratamiento. La importancia de ello es que incluir la evaluación de la calidad del sueño e identificar a los malos dormidores de forma precoz, permitiría identificar poblaciones vulnerables -con una probabilidad mayor de abandonar el tratamiento- en el contexto de la práctica clínica ambulatoria. Asimismo, la mala calidad del sueño moderada/severa afecta notoriamente la calidad de vida y se requiere evaluación médica especializada; por lo cual su detección permitiría derivar a los pacientes para un manejo adecuado y evitar complicaciones subsecuentes 61,62. Por ello, se recomienda que se investigue y se recolecte información que permita evaluar la calidad del sueño en estos pacientes con los fines de intervenir tempranamente los efectos asociados, como el impacto en la adherencia al TARGA.

En conclusión, se encontró asociación entre la calidad de sueño y la adherencia al tratamiento antirretroviral de alta actividad en el grupo en estudio. Finalmente, se recomienda un abordaje integral de los pacientes en consulta ambulatoria para la identificación de factores que afecten su adherencia al tratamiento y de esta forma mejorar su calidad de sueño, que podría ser un predictor o indicador precoz de un potencial abandono al tratamiento.

\section{Resumo}

A fim de avaliar se a associação entre a má qualidade do sono e adesão à terapia antirretroviral (TARV) em pessoas com infecção pelo HIVIAIDS, realizamos um estudo analítico de corte transversal que incluiu 389 pacientes peruanos em TARV. A má qualidade do sono foi medida com a escala Índice de Qualidade de Sono de Pittsburgh (IQSP) e a aderência com o CEATHIV (adaptação peruana). Foi realizado um modelo linear generalizado da família Poisson com padrão de erros robustos, para estimar as razões de prevalência e IC95\%. No nível cru, a má qualidade do sono leve, moderada e grave foram associadas com adesão inade- quada. Quando fizemos o ajuste para as variáveis associadas na análise bivariada ou variáveis teoricamente associadas à adesão, somente a má qualidade moderada/grave manteve-se associada ( $R P=1,34$; IC95\%: 1,171,54 e $R P=1,34$; IC95\%: 1,16-1,57; respectivamente). Concluímos que a má qualidade do sono moderadal grave está independentemente associada com a adesão a TARV. Avaliar a qualidade do sono pode ser útil na avaliação global do paciente com HIV.

Transtornos do Sono; HIV; Terapia Antirretroviral de Alta Atividade 


\section{Colaboradores}

J. R. Tello-Velásquez y B. E. Díaz-Llanes contribuyó en el diseñó, el análisis y redacción el artículo, coordinó la encuesta de la que se obtuvo la información para este análisis y revisó y aprobó la versión final. E. MezonesHolguín contribuyó con el diseño, el análisis y redacción el artículo, revisó y aprobó la versión final. A. J. Rodríguez-Morales, C. Huamaní y A. V. Hernández participó en el análisis, revisó y aprobó la versión final. J. Arévalo-Abanto contribuyó na avaliación del instrumento para análisis, na revisión y aprobación de la versión final.

\section{Agradecimientos}

Los autores de este trabajo agradecen el apoyo del Servicio de Infectología del Hospital Nacional Dos de Mayo y a la Universidad Peruana de Ciencias Aplicadas. Se agradece la gentil participación de los pacientes en esta investigación.

\section{Referencias}

1. Murray CJ, Ortblad KF, Guinovart C, Lim SS, Wolock TM, Roberts DA, et al. Global, regional, and national incidence and mortality for HIV, tuberculosis, and malaria during 1990-2013: a systematic analysis for the Global Burden of Disease Study 2013. Lancet 2014; 384:1005-70.

2. Larson HJ, Bertozzi S, Piot P. Redesigning the AIDS response for long-term impact. Bull World Health Organ 2011; 89:846-52.

3. Vella S, Schwartländer B, Sow SP, Eholie SP, Murphy RL. The history of antiretroviral therapy and of its implementation in resource-limited areas of the world. AIDS 2012; 26:1231-41.

4. Joint United Nations Program on HIV/AIDS. HIV and AIDS estimates (2013). http://www.unaids. org/en/regionscountries/countries/peru/ (accedido el 20/Sep/2014)

5. Teva I, Bermúdez MP, Ramiro MT, Buela-Casal G. Situación epidemiológica actual del VIH/SIDA en Latinoamérica en la primera década del siglo XXI. Análisis de las diferencias entre países. Rev Med Chil 2012; 140:50-8.
6. Gupta RK, Wainberg MA, Brun-Vezinet F, Gatel JM, Albert J, Sönnerborg A, et al. Oral antiretroviral drugs as public health tools for HIV prevention: global implications for adherence, drug resistance, and the success of HIV treatment programs. J Infect Dis 2013; 207 Suppl 2:S101-6.

7. Saldarriaga-Arenas PA, Rodríguez-Morales AJ. Epidemiology of opportunistic diseases in AIDS patients from Pereira municipality, Colombia, 20102011. J Infect Public Health 2013; 6:496-8.

8. Pulvirenti JJ. Inpatient care of the HIV infected patient in the highly active antiretroviral therapy (HAART) era. Curr HIV Res 2005; 3:133-45.

9. Fauci AS, Marston HD. Achieving an AIDS-free world: science and implementation. Lancet 2013; 382:1461-2.

10. Barth RE, van der Meer JTM, Hoepelman AIM, Schrooders PA, van de Vijver DA, Geelen SPM, et al. Effectiveness of highly active antiretroviral therapy administered by general practitioners in rural South Africa. Eur J Clin Microbiol Infect Dis 2008; 27:977-84. 
11. Farnham PG, Gopalappa C, Sansom SL, Hutchinson AB, Brooks JT, Weidle PJ, et al. Updates of lifetime costs of care and quality-of-life estimates for HIV-infected persons in the United States: late versus early diagnosis and entry into care. J Acquir Immune Defic Syndr 2013; 64:183-9.

12. Kato M, Granich R, Bui DD, Tran HV, Nadol P, Jacka D, et al. The potential impact of expanding antiretroviral therapy and combination prevention in Vietnam: towards elimination of HIV transmission. J Acquir Immune Defic Syndr 2013; 63: e142-9.

13. Bhatia R, Ryscavage P, Taiwo B. Accelerated aging and human immunodeficiency virus infection: emerging challenges of growing older in the era of successful antiretroviral therapy. J Neurovirol 2012; 18:247-55,

14. Deeks SG, Lewin SR, Havlir DV. The end of AIDS: HIV infection as a chronic disease. Lancet 2013; 382:1525-33.

15. Achappa B, Madi D, Bhaskaran U, Ramapuram JT, Rao S, Mahalingam S. Adherence to antiretroviral therapy among people living with HIV. N Am J Med Sci 2013; 5:220-3.

16. Aragonés C, Sánchez L, Campos JR, Pérez J. Antiretroviral therapy adherence in persons with HIV/ AIDS in Cuba. MEDICC Rev 2011; 13:17-23.

17. Alave J, Paz J, González E, Campos M, Rodríguez M, Willig J, et al. Factores asociados a falla virológica en pacientes infectados con VIH que reciben terapia anti-retroviral en un hospital público del Perú. Rev Chilena Infectol 2013; 30:42-8.

18. Elbirt D, Asher I, Mahlev-Guri K, Bezalel-Rozenberg S, Werner B, Cohen Y, et al. Direct monthly highly active antiretroviral therapy supply: a method to increase patient's adherence and outcome. Experience of one AIDS center in Israel. Int J STD AIDS 2013; 25:579-86.

19. Ortego C, Huedo-Medina TB, Llorca J, Sevilla L, Santos P, Rodríguez E, et al. Adherence to highly active antiretroviral therapy (HAART): a metaanalysis. AIDS Behav 2011; 15:1381-96.

20. Shin S, Muñoz M, Espiritu B, Zeladita J, Sanchez E, Callacna M, et al. Psychosocial impact of poverty on antiretroviral nonadherence among HIV-TB coinfected patients in Lima, Peru. J Int Assoc Physicians AIDS Care (Chic) 2008; 7:74-81.

21. Nel A, Kagee A. Common mental health problems and antiretroviral therapy adherence. AIDS Care 2011; 23:1360-5.

22. Hendershot CS, Stoner SA, Pantalone DW, Simoni JM. Alcohol use and antiretroviral adherence: review and meta-analysis. J Acquir Immune Defic Syndr 2009; 52:180-202.

23. Grierson J, Koelmeyer RL, Smith A, Pitts M. Adherence to antiretroviral therapy: factors independently associated with reported difficulty taking antiretroviral therapy in a national sample of HIVpositive Australians. HIV Med 2011; 12:562-9.

24. Springer SA, Dushaj A, Azar MM. The impact of DSM-IV mental disorders on adherence to combination antiretroviral therapy among adult persons living with HIV/AIDS: a systematic review. AIDS Behav 2012; 16:2119-43.
25. Malow R, Dévieux JG, Stein JA, Rosenberg R, JeanGilles M, Attonito J, et al. Depression, substance abuse and other contextual predictors of adherence to antiretroviral therapy (ART) among Haitians. AIDS Behav 2013; 17:1221-30.

26. Nakimuli-Mpungu E, Bass JK, Alexandre P, Mills EJ, Musisi S, Ram M, et al. Depression, alcohol use and adherence to antiretroviral therapy in subSaharan Africa: a systematic review. AIDS Behav 2012; 16:2101-18.

27. Memiah P, Shumba C, Etienne-Mesubi M, Agbor S, Hossain MB, Komba P, et al. The effect of depressive symptoms and CD4 count on adherence to highly active antiretroviral therapy in sub-Saharan Africa. J Int Assoc Provid AIDS Care 2013; 13: 346-52.

28. Azar MM, Springer SA, Meyer JP, Altice FL. A systematic review of the impact of alcohol use disorders on HIV treatment outcomes, adherence to antiretroviral therapy and health care utilization. Drug Alcohol Depend 2010; 112:178-93.

29. Tran BX, Nguyen LT, Do CD, Nguyen QL, Maher RM. Associations between alcohol use disorders and adherence to antiretroviral treatment and quality of life amongst people living with HIV/ AIDS. BMC Public Health 2014; 14:27.

30. Sreenivasan S, Dasegowda V. Adverse effects after HAART Initiation in resource-limited settings: a prospective study from Mysore, India. J Infect Dev Ctries 2010; 4:750-3.

31. Lenzi L, Wiens A, Pontarolo R. Evaluation of adverse events associated with antiretroviral therapy and the relationship to treatment adherence. Int J Clin Pharmacol Ther 2013; 51:141-6.

32. Franke MF, Murray MB, Muñoz M, HernándezDíaz S, Sebastián JL, Atwood S, et al. Food insufficiency is a risk factor for suboptimal antiretroviral therapy adherence among HIV-infected adults in urban Peru. AIDS Behav 2011; 15:1483-9.

33. Obirikorang C, Selleh PK, Abledu JK, Fofie CO. Predictors of adherence to antiretroviral therapy among HIV/AIDS patients in the upper West Region of Ghana. ISRN AIDS 2013; 2013:873939.

34. Taibi DM. Sleep disturbances in persons living with HIV. J Assoc Nurses AIDS Care 2013; 24(1 Suppl):S72-85.

35. Wibbeler T, Reichelt D, Husstedt I-W, Evers S. Sleepiness and sleep quality in patients with HIV infection. J Psychosom Res 2012; 72:439-42.

36. Junqueira P, Bellucci S, Rossini S, Reimão R. Women living with HIV/AIDS: sleep impairment, anxiety and depression symptoms. Arq Neuropsiquiatr 2008; 66:817-20.

37. Bialer PA, Wallack JJ, Prenzlauer SL, Bogdonoff L, Wilets I. Psychiatric comorbidity among hospitalized AIDS patients vs. non-AIDS patients referred for psychiatric consultation. Psychosomatics 1996; 37:469-75

38. Babson KA, Heinz AJ, Bonn-Miller MO. HIV medication adherence and HIV symptom severity: the roles of sleep quality and memory. AI5DS Patient Care STDs 2013; 27:544-52.

39. Saberi P, Neilands TB, Johnson MO. Quality of sleep: associations with antiretroviral nonadherence. AIDS Patient Care STDs 2011; 25:517-24. 
40. Saberi P, Comfort M, Sheon N, Johnson MO. Qualitative study of the quality of sleep in marginalized individuals living with HIV. Patient Prefer Adherence 2013; 7:499-507.

41. Phillips KD, Moneyham L, Murdaugh C, Boyd MR, Tavakoli A, Jackson K, et al. Sleep disturbance and depression as barriers to adherence. Clin Nurs Res 2005; 14:273-93.

42. Escobar-Córdoba F, Eslava-Schmalbach J. Validación colombiana del índice de calidad de sueño de Pittsburgh. Rev Neurol 2005; 40:150-5.

43. Buysse DJ, Reynolds 3rd CF, Monk TH, Berman SR, Kupfer DJ. The Pittsburgh Sleep Quality Index: a new instrument for psychiatric practice and research. Psychiatry Res 1989; 28:193-213.

44. Prieto-Rincón D, Echeto-Inciarte S, FaneiteHernández P, Inciarte-Mundo J, Rincón-Prieto C, Bonilla E. Calidad del sueño en pacientes psiquiátricos hospitalizados. Invest Clin 2006; 47:5-16.

45. Remor E. Valoración de la adhesión al tratamiento antirretroviral en pacientes VIH+. Psicothema 2002; 14:262-7.

46. Tafur-Valderrama E, Ortiz C, Alfaro CO, GarcíaJiménez E, Faus MJ. Adaptación del "Cuestionario de Evaluación de la adhesión al Tratamiento antirretroviral" (CEAT-VIH) para su uso en Perú. Ars Pharm 2008; 49:183-98.

47. Reis AC, Lencastre L, Guerra MP, Remor E. Adaptação portuguesa do questionário para a avaliação da adesão ao tratamento anti-retrovírico VIH (CEAT-VIH). Psicol Saúde Doenças 2009; 10:175-91.

48. Remor E. Systematic review of the psychometric properties of the questionnaire to evaluate the adherence to HIV therapy (CEAT-VIH). Patient 2013; 6:61-73.

49. Calderón R, Quiroz A, Rosales E, Rey de Castro J. Calidad de sueño en pobladores de una comunidad andina a 3200 msnm en Perú: Ancash-Perú. Rev Méd Hered 2010; 21:65-9.

50. Rey de Castro J, Rosales-Mayor E. Monitoreo del sueño en conductores de ómnibus y camiones: factor relevante a considerar para la renovación de la licencia de conducir. Rev Peru Med Exp Salud Publica 2010; 27:260-6.

51. Tello Rodriguez T, Varela Pinedo L, Ortiz Saavedra PJ, Chávez Jimeno H, Revoredo Gonzáles C. Calidad del sueño, somnolencia diurna e higiene del sueño en el Centro del Adulto Mayor Mirones, EsSalud, Lima, Perú. Acta Méd Perú 2009; 26:22-6.

52. Díaz-Llanes BE, Tello-Velásquez JR, MezonesHolguin E, Arévalo J, Rodríguez-Morales AJ. Mala calidad de sueño en pacientes que reciben terapia anti-retroviral de gran actividad: ¿necesidad de evaluación en la práctica ambulatoria? Rev Chilena Infectol 2012; 29:478.

53. Shrestha BM. The Declaration of Helsinki in relation to medical research: historical and current perspectives. J Nepal Health Res Counc 2012; 10:254-7.

54. Kenedi CA, Goforth HW. A systematic review of the psychiatric side-effects of efavirenz. AIDS Behav 2011; 15:1803-18.
55. Ferreira LTK, Ceolim MF. Qualidade do sono em portadores do vírus da imunodeficiência humana. Rev Esc Enferm USP 2012; 46:892-9.

56. Peltzer K, Friend-du Preez N, Ramlagan S, Anderson J. Antiretroviral treatment adherence among HIV patients in KwaZulu-Natal, South Africa. BMC Public Health 2010; 10:111.

57. Shaahu VN, Lawoyin TO, Sangowawa AO. Adherence to highly active antiretroviral therapy (HAAT) at a Federal Medical Centre. Afr J Med Med Sci 2008; 37:29-36.

58. Uzochukwu BSC, Onwujekwe OE, Onoka AC, Okoli C, Uguru NP, Chukwuogo OI. Determinants of non-adherence to subsidized anti-retroviral treatment in southeast Nigeria. Health Policy Plan 2009; 24:189-96.

59. Kiwuwa-Muyingo S, Walker AS, Oja H, Levin J, Miiro G, Katabira E, et al. The impact of first year adherence to antiretroviral therapy on long-term clinical and immunological outcomes in the DART trial in Uganda and Zimbabwe. Trop Med Int Health 2012; 17:584-94.

60. Charurat M, Oyegunle M, Benjamin R, Habib A, Eze E, Ele P, et al. Patient retention and adherence to antiretrovirals in a large antiretroviral therapy program in Nigeria: a longitudinal analysis for risk factors. PLoS One 2010; 5:e10584.

61. Boarts JM, Sledjeski EM, Bogart LM, Delahanty DL. The differential impact of PTSD and depression on HIV disease markers and adherence to HAART in people living with HIV. AIDS Behav 2006; 10:253-61.

62. Neuman MG, Schneider M, Nanau RM, Parry C. Alcohol consumption, progression of disease and other comorbidities, and responses to antiretroviral medication in people living with HIV. AIDS Res Treat 2012; 2012:751827.

63. Bonilla J, Bernal G, Santos A, Santos D. A revised Spanish version of the Beck Depression Inventory: psychometric properties with a Puerto Rican sample of college students. J Clin Psychol 2004; 60:119-30.

64. Buckley TM, Schatzberg AF. On the interactions of the hypothalamic-pituitary-adrenal (HPA) axis and sleep: normal HPA axis activity and circadian rhythm, exemplary sleep disorders. J Clin Endocrinol Metab 2005; 90:3106-14.

65. Rothman KJ, Greenland S, Lash TL. Modern epidemiology. 3rd Ed. Philadelphia: Lippincott Williams \&Wilkins; 2008.

66. Szklo M, Nieto FJ. Epidemiology: beyond the basics. Burlington: Jones \& Bartlett Publishers; 2012.

67. Ten Have TR, Joffe MM. A review of causal estimation of effects in mediation analyses. Stat Methods Med Res 2012; 21:77-107.

68. Pearl J. An introduction to causal inference. Int J Biostat 2010; 6:7.

Recibido el 21/Ene/2014

Versión final presentada el 24/Nov/2014

Aprobado el 01/Dic/2014 